\title{
The Effect of Wasabi Rhizome Extract on Atopic Dermatitis-Like Symptoms in HR-1 Hairless Mice
}

\author{
Masashi NAGAI and Isao OKUNISHI \\ Kinjirushi Co., Ltd., 2-61, Yahata-hontori, Nakagawa-ku, Nagoya 454-8526, Japan
}

(Received September 10, 2008)

\begin{abstract}
Summary We investigated the effect of wasabi rhizome extract on atopic dermatitis (AD) model mice. The wasabi extract was fed to the HR-1 hairless mice, which develop AD-like symptoms with a special diet (HR-AD diet). The extract was expected to reduce the symptoms induced. Wasabi rhizome-containing HR-AD diet (5\% and $10 \%)$ reduced the scratching behavior, and the $10 \%$ wasabi rhizome HR-AD diet significantly reduced scratching behavior on days 28, 35 and 42. Plasma components (histamine, eotaxin, IgE and thymus and activation-regulated chemokine (TARC)) were decreased in the $10 \%$ wasabi rhizome HR-AD diet. In histopathological examinations (toluidine blue (T.B.), major basic protein (MBP), CD4, IL-4, IL-5, eotaxin, TARC and IgE), the wasabi rhizome-containing HR-AD diet ( $5 \%$ and $10 \%)$ significantly reduced the number of positive stained cells. These results suggested that the wasabi rhizome extract improved the AD-like symptoms of HR-1 hairless mice.
\end{abstract}

Key Words wasabi, 6-methylsulfinylhexyl isothiocyanate, atopic dermatitis, HR-1 hairless mice

Atopic dermatitis (AD) is a chronic dermatologic disease characterized by itchy skin (1). Histopathologically, $\mathrm{AD}$ exhibits an inflammation with thickened epidermis, and infiltration of a lymphocyte, eosinophil and mast cell to the corium. For a diagnosis of AD, several markers were studied. Serum IgE and histamine levels are elevated in patients with $\mathrm{AD}(2,3)$. But the relationship between IgE and clinical disease is not always exclusive. It was reported that the density of the mast cells of skin increased in AD-model animals $(4,5)$. Thymus and activation-regulated chemokine (TARC) is the CC chemokine that is expressed in keratinocytes. The induction of TARC production plays an important role in the lesional skin through the chemokine receptor $\mathrm{CCR} 4^{+}$/ cutaneous lymphocyte-associated antigen (CLA) ${ }^{+}$Th2type lymphocytes in AD (6). Dworzak et al. reported that patients with severe $\mathrm{AD}$ had a significantly expanded proportion of $\mathrm{CLA}^{+} / \mathrm{CD}^{+}{ }^{+} \mathrm{T}$ cells compared with control subjects (7). Eotaxin is chemotactic factor that is produced by a stimulus of IL-4 from fibroblasts (8). Skin lesions are caused by IL-5 or eotaxin-mediated eosinophilic infiltration into the skin (9). Major basic protein (MBP) is the basic protein in eosinophil granules, and possesses cellular cytotoxicity (10). Eosinophils are involved in the inflammatory response in $\mathrm{AD}$ by the release of MBP. In patients with $\mathrm{AD}$, chemical mediators, such as TARC, eotaxin and MBP, exhibit a high value, and are well correlated with clinical presentation (11-14).

Wasabi (Wasabia japonica (Miq) Matsumura) is a plant

E-mail: m-nagai@kinjirushi.co.jp of Japanese origin, and it belongs to the Brassicaceae family. Wasabi has been used in Japanese life as a spice from ancient times. It is known that wasabi inhibits proliferation of bacteria, and this has been studied for many years $(15,16)$. It has been reported that wasabi extract and fragrance ingredient (6-methylsulfinylhexyl isothiocyanate; 6-MSITC) had a repressive effect on diabetic nephropathy in type 2 diabetic mice (17), and a platelet aggregation-inhibitory activity in human platelets (18). In a recent study, Uto et al. reported that 6MSITC suppressed inducible nitric oxide synthase (iNOS) production of RAW264 cells, and the suppressive effects depended on inhibition in the MAPK signaling pathways (19). The expression of iNOS was upregulated in the dermal lesions of AD-model mice (20). Taniuchi et al. reported that the disease severity in patients with $\mathrm{AD}$ was significantly correlated with serum nitrate levels (21). These results suggested that wasabi ingredients had the possibility to improve AD.

In this study, the wasabi extract was fed to the HR-1 hairless mice, which develop AD-like symptoms with a special diet. The repressing effect on scratching behavior and the reduction effects on the histopathological index were investigated.

\section{Materials and Methods}

Wasabi rhizome extract. Freeze-crushed wasabi rhizome $(8.0 \mathrm{~kg})$ and distilled water $(8.0 \mathrm{~kg})$ were mixed and incubated for $3 \mathrm{~h}$ in $37^{\circ} \mathrm{C}$. The mixture was moved to a closed container and volatile isothiocyanates were removed by decompression processing. Fifty percent ethanol $(40 \mathrm{~kg})$ was added to the mixture and it was 
Table 1. Ingredients of the wasabi rhizome extract.

\begin{tabular}{ll}
\hline & Ingredients (per kg) \\
\hline Energy & $14,500 \mathrm{~kJ}$ \\
Moisture & $55 \mathrm{~g}$ \\
Crude protein & $144 \mathrm{~g}$ \\
Crude fat & $5 \mathrm{~g}$ \\
Carbohydrate & $713 \mathrm{~g}$ \\
Crude ash & $83 \mathrm{~g}$ \\
$\mathrm{Na}$ & $3,990 \mathrm{mg}$ \\
$\mathrm{Mg}$ & $3,680 \mathrm{mg}$ \\
Zn & $25.8 \mathrm{mg}$ \\
6-MSITC & $1,400 \mathrm{mg}$ \\
Allyl isothiocyanate & $0 \mathrm{mg}$
\end{tabular}

Analysis method of each ingredient is as follows. Energy: protein $(\mathrm{g}) \times 16.7 \mathrm{~kJ}+$ fat $(\mathrm{g}) \times 37.7 \mathrm{~kJ}+$ carbohydrate $(\mathrm{g})$ $\times 16.7 \mathrm{~kJ}$, moisture: air oven method, crude protein: Kjeldahl method, crude fat: acid hydrolysis method, carbohydrate: $1,000-($ moisture $(\mathrm{g})+$ protein $(\mathrm{g})+$ fat $(\mathrm{g})+$ crude ash $(\mathrm{g})$ ), crude ash: direct ashing method, Na: atomic absorption spectrometry, $\mathrm{Mg}$ and $\mathrm{Zn}$ : inductively coupled plasma spectrometry, 6-MSITC and allyl isothiocyanate: gas chromatography.

stirred at room temperature for $1 \mathrm{~h}$. After centrifuge separation, it was filtered using diatomite and $39 \mathrm{~kg}$ of extractive liquid was obtained. The extractive liquid was concentrated using a rotary evaporator, and spray dried using $370 \mathrm{~g}$ of dextrin (Matsutani Chemical Industry Co., Ltd., Hyogo, Japan). Finally, $650 \mathrm{~g}$ of powdered wasabi rhizome extract was obtained. The ingredients of the wasabi rhizome extract are shown in Table 1. 6MSITC was detected in the wasabi rhizome extract, but other isothiocyanates weren't detected.

Animals. Male HR-1 hairless mice ( 4 wk old) were obtained from Hoshino Laboratory Animals (Saitama, Japan). HR-1 hairless mice were fed a special diet to develop AD-like symptoms. The detailed ingredients of the diet have been described (22). The experiment obeyed the "Guidelines for proper conduct of animal experiments" of the Science Council of Japan, and was performed ethically.

Care conditions. Male HR-1 hairless mice (5 wk old) were sorted by stratified randomization based on body weight before day one of the feeding. Mice were housed in a conventional animal room, under controlled temperature $\left(22 \pm 3^{\circ} \mathrm{C}\right)$, humidity $(50 \pm 20 \%)$ and ventilation $(13-17 / \mathrm{h})$ with lights on from 08:00 to 20:00. They were housed in polycarbonate cages separately, and given tap water freely. They were fed a normal diet (Labo MR stock, Nosan Corp., Yokohama, Japan) or a HR-AD diet (HR-AD manufactured diet, Nosan Corp.) or a diet with wasabi rhizome extract $(5 \%$ or $10 \%)$ added to the HR-AD manufactured diet, with free feeding. The wasabi rhizome extract and the HR-AD diet were mixed once a week. About $83 \%$ of 6 -MSITC in HR$\mathrm{AD}+$ wasabi diet remained after $1 \mathrm{wk}$. On day 42 , plasma samples were taken for hematological examination, and dorsal skin samples were isolated and fixed by
$10 \%$ neutral formalin. The energy content of each diet was calculated by the value indicated on the catalog of the diet and the measurement of wasabi rhizome extract.

Measurement of scratching behavior. The scratching behavior of the mice was analyzed and recognized at three time points: 28,35 and $42 \mathrm{~d}$ after starting the special diet. Mice were videotaped for $30 \mathrm{~min}$, and the frequency of scratching was counted retrospectively at sequential 5-min intervals (23). Scratching frequency was defined as scratching the neck with the hind limbs. The cumulative scratching frequency every 5 min was calculated.

Hematology. In the plasma samples, histamine (Histamine ELISA, IBL Co., Ltd., Gunma, Japan), eotaxin (Quantikine $^{\circledR}$ Immunoassay Eotaxin, R\&D Systems, Inc., Minneapolis, USA), IgE (Mouse IgE ELISA Quantitation Kit, Bethyl Laboratories, Inc., Montgomery, USA), TARC (Quantikine ${ }^{\circledR}$ Immunoassay CCL17/TARC, R\&D Systems, Inc.) were analyzed according to the manual of the kit.

Skin histopathology. Skin samples were stained with several methods and the positive stained cells counted in the view area of the microscope $(\times 400)$. For the examination of mast cells, the tissues were stained with toluidine blue (T.B.) (Wako Pure Chemical Industries, Ltd., Osaka, Japan). Immunohistological indexes were detected using anti-mouse antibodies. Antibodies such as MBP (Biogenesis Inc., Poole, UK), CD4 (Santa Cruz Biotechnology, Inc., California, USA), IL-4 (Santa Cruz Biotechnology), IL-5 (Santa Cruz Biotechnology), eotaxin (Chemicon International Inc., California, USA), TARC (Santa Cruz Biotechnology) and IgE (Biogenesis Inc.) were used.

Statistical analysis. Results were expressed as the mean \pm SE for each group in diet intake, body weight and scratching behavior, and as the mean \pm SD for each group in the plasma components and the histopathological indexes. For body weight and diet intake, the significant difference between normal diet and the HR-AD diet was determined using the Student's $t$-test if the sequence was homoscedastic, or Aspin-Welch's $t$-test when it was heteroscedastic, after doing the F-test. The variance between the HR-AD diet and $\mathrm{HR}-\mathrm{AD}+5 \%$ or $10 \%$ of wasabi rhizome extract diet was tested by the Bartlett test. When it was homoscedastic, they were tested by one-way ANOVA and when heteroscedastic, they were tested by the Kruskal-Wallis test. When a variance was significant, the significant difference was determined using Tukey's test. For scratching behavior, the significant difference between the normal diet and the HR-AD diet, HR-AD diet and HR-AD $+5 \%$ or $10 \%$ of wasabi rhizome extract diet was determined using Student's t-test when it was homoscedastic, and AspinWelch's $t$-test when it was heteroscedastic, after doing the F-test. For the hematological examination and histopathological examination, the significant difference of each diet was determined using one-way ANOVA. Values of $p<0.05$ were considered to be statistically significant in the F-test, Bartlett test, one-way ANOVA and 
Table 2. Time-course changes in diet intake of HR-1 hairless mice (g).

\begin{tabular}{|c|c|c|c|c|c|c|c|}
\hline & \multicolumn{7}{|c|}{ Time (d) } \\
\hline & 0 & 7 & 14 & 21 & 28 & 35 & 42 \\
\hline Normal & $4.6 \pm 0.3_{*}$ & $5.5 \pm 0.2_{*}$ & $5.7 \pm 0.3_{* *}$ & $6.1 \pm 0.3_{* *}$ & $6.5 \pm 0.2$ & $6.8 \pm 0.3_{* *}$ & $6.5 \pm 0.2_{* *}$ \\
\hline HR-AD & $3.8 \pm 0.2^{\rfloor}$ & $4.1 \pm 0.3^{\rfloor}$ & $4.4 \pm 0.2^{\rfloor}$ & $4.6 \pm 0.1^{\rfloor}$ & $4.4 \pm 0.4$ & $3.7 \pm 0.3]$ & $3.6 \pm 0.5^{\rfloor}$ \\
\hline HR-AD $+5 \%$ wasabi & $3.9 \pm 0.1$ & $4.3 \pm 0.2$ & $4.8 \pm 0.2$ & $5.0 \pm 0.2$ & $5.7 \pm 0.3^{\rfloor}$ & $5.2 \pm 0.3^{]}$ & $5.2 \pm 0.5$ \\
\hline HR-A $+10 \%$ wasabi & $3.6 \pm 0.2$ & $3.9 \pm 0.1$ & $4.3 \pm 0.1$ & $4.5 \pm 0.2$ & $5.4 \pm 0.2$ & $5.2 \pm 0.3-$ & $5.1 \pm 0.6$ \\
\hline
\end{tabular}

The diet intake of the HR-1 mice was analyzed and recorded every $7 \mathrm{~d}$, after starting the special diet. Values given are the mean \pm SE of 8 animals. $t$-test: ${ }^{*} p<0.05,{ }^{* *} p<0.01$. Tukey's test: ${ }^{\#} p<0.05,{ }^{\# \#} p<0.01$.

Table 3. Time-course changes in body weight of HR-1 hairless mice (g).

\begin{tabular}{|c|c|c|c|c|c|c|c|c|}
\hline & \multirow{2}{*}{$\begin{array}{l}\text { Group } \\
\text { sorting }\end{array}$} & \multicolumn{7}{|c|}{ Time (d) } \\
\hline & & 0 & 7 & 14 & 21 & 28 & 35 & 42 \\
\hline Normal & $19.4 \pm 0.4$ & $19.8 \pm 0.4$ & $23.9 \pm 0.3$ & $26.0 \pm 0.3$ & $27.3 \pm 0.4$ & $28.5 \pm 0.4$ & $29.2 \pm 0.4$ & $29.9 \pm 0.4$ \\
\hline HR-AD & $19.2 \pm 0.4$ & $18.1 \pm 0.5^{\lrcorner}$ & $22.7 \pm 0.5^{\rfloor}$ & $24.8 \pm 0.6$ & $26.4 \pm 0.6$ & $26.6 \pm 1.1$ & $27.6 \pm 0.7$ & $27.5 \pm 0.9$ \\
\hline HR-AD $+5 \%$ wasabi & $19.1 \pm 0.4$ & $18.6 \pm 0.5$ & $23.3 \pm 0.5$ & $25.8 \pm 0.6$ & $27.8 \pm 0.7$ & $29.4 \pm 0.8$ & $29.9 \pm 0.8$ & $30.4 \pm 0.7$ \\
\hline HR-A $+10 \%$ wasabi & $19.1 \pm 0.4$ & $18.2 \pm 0.3$ & $22.6 \pm 0.3$ & $24.9 \pm 0.4$ & $26.9 \pm 0.4$ & $28.5 \pm 0.4$ & $29.5 \pm 0.3$ & $29.8 \pm 0.3$ \\
\hline
\end{tabular}

The body weight of the mice was analyzed and recorded every $7 \mathrm{~d}$, after starting the special diet. Mice were sorted by stratified randomization based on body weight before day one of the feeding. Values given are the mean \pm SE of 8 animals. $t$-test: ${ }^{*} p<0.05$. Tukeyfs test: ${ }^{\#} p<0.05$.

Kruskal-Wallis test. Values of $p<0.05$ and $p<0.01$ were considered to be statistically significant in the Student's $t$-test and Tukey's test.

\section{Results and Discussion}

Diet intake and body weight

The intake of normal diet-fed mice exhibited an increase over the time-course (Table 2). The intake of HR-AD-fed mice was significantly less than that of normal diet-fed mice. The intake of $\mathrm{HR}-\mathrm{AD}+5 \%$ wasabi-fed mice exhibited a significantly high value on days 28 and 35 compared with that of the HR-AD diet-fed mice. The intake of HR-AD $+10 \%$ wasabi-fed mice exhibited a significantly high value on day 35 compared with that of the HR-AD diet-fed mice. Throughout the measurement period, no statistically significant difference was seen between normal diet-fed mice and HR$\mathrm{AD}+$ wasabi-fed mice.

The body weight was increased smoothly in normal diet-fed mice (Table 3). The body weight of HR-AD-fed mice was less than that of the normal diet-fed mice, especially on days 0,7 and 42 . There was no significant difference in body weight among normal diet-fed mice and $\mathrm{HR}-\mathrm{AD}+5 \%$ wasabi-fed mice. On day 42 , the body weight of $\mathrm{HR}-\mathrm{AD}+5 \%$ wasabi-fed mice indicated a high value compared with that of HR-AD-fed mice. There

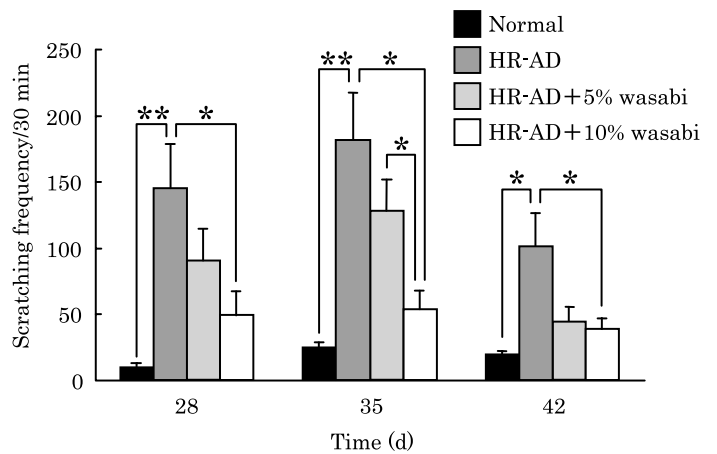

Fig. 1. Effects of wasabi rhizome extract on the scratching behavior in normal diet-fed mice, HR-AD diet-fed mice, HR-AD $+5 \%$ wasabi diet-fed mice and HR$\mathrm{AD}+10 \%$ wasabi diet-fed mice. Values given are the mean \pm SE of 8 animals. One-way ANOVA: ${ }^{*} p<0.05$, ${ }^{* *} p<0.01$.

was no significant difference in body weight among normal diet-fed mice and HR-AD $+10 \%$ wasabi-fed mice.

The energy contents of the normal diet, HR-AD diet, HR-AD $+5 \%$ wasabi diet and HR-AD $+10 \%$ wasabi diet were estimated at $10,850,14,810,14,800$ and 14,780 $\mathrm{kJ} / \mathrm{kg}$, respectively. The accumulations of energy intake on measurement days (days $0,7,14,21,28,35$ and 
A

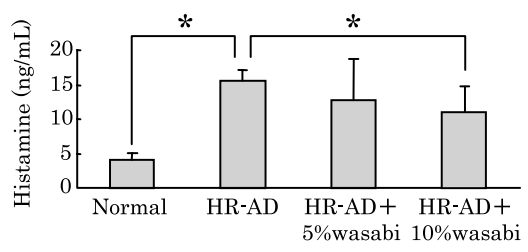

$\mathrm{C}$

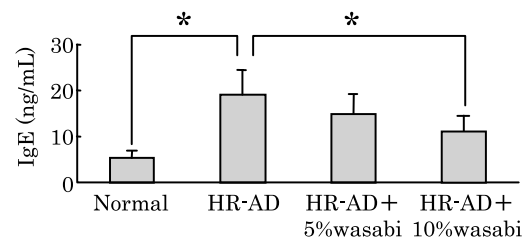

B

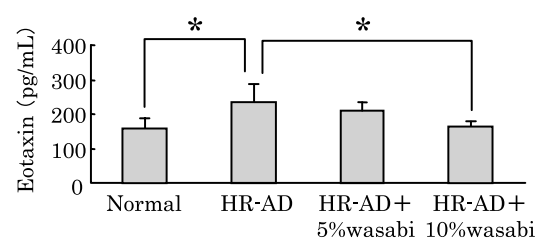

$\mathrm{D}$

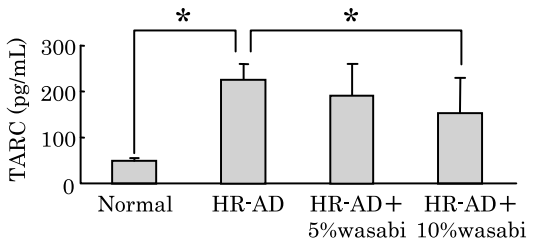

Fig. 2. Hematological examination of plasma of HR-1 mice fed a normal diet, HR-AD diet, HR-AD+5\% wasabi diet and HR-AD $+10 \%$ wasabi diet, on day 42. Charts show histamine (A), eotaxin (B), IgE (C) and TARC (D). Values given are the mean \pm SD of 8 animals. One-way ANOVA: ${ }^{*} p<0.05$.
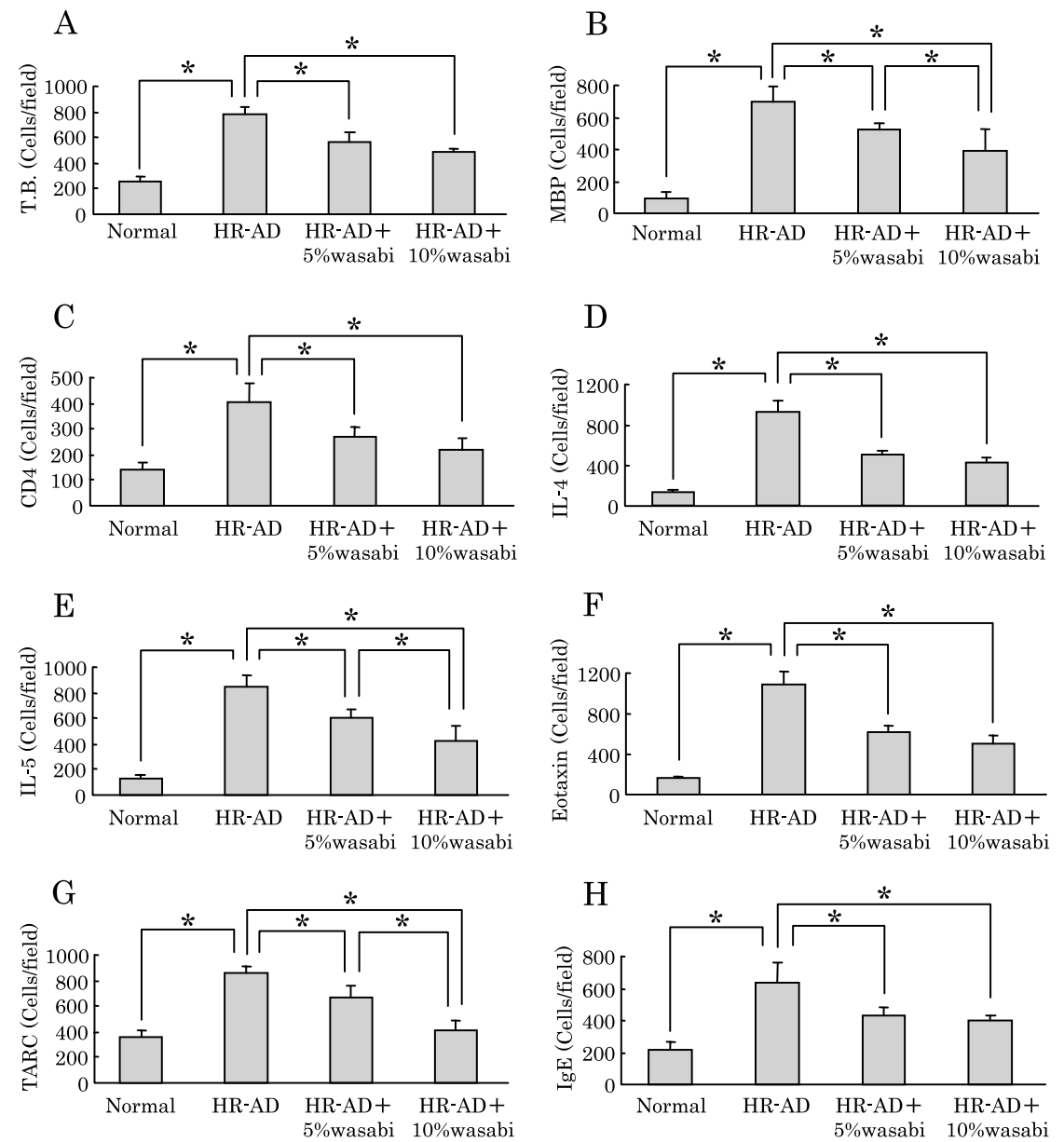

Fig. 3. Histopathological examination of skin from HR-1 mice fed a normal diet, HR-AD diet, HR-AD+5\% wasabi diet and HR-AD $+10 \%$ wasabi diet, on day 42. Charts show T.B. (A), MBP (B), CD4 (C), IL-4 (D), IL-5 (E), Eotaxin (F), TARC (G) and $\operatorname{IgE}(\mathrm{H})$. Values given are the mean \pm SD of 8 animals. One-way ANOVA: ${ }^{*} p<0.05$.

42) were estimated at $453.7 \pm 15.0 \mathrm{~kJ}$ on the normal diet, $423.6 \pm 15.9 \mathrm{~kJ}$ on the HR-AD diet, $502.3 \pm$ $20.2 \mathrm{~kJ}$ on the HR-AD+5\% wasabi diet and $473.5 \pm$ $15.1 \mathrm{~kJ}$ on the HR-AD $+10 \%$ wasabi diet (mean $\pm \mathrm{SE}$ ). The respective $\mathrm{HR}-\mathrm{AD}+$ wasabi diet had an energy intake higher than the HR-AD diet $(p<0.05)$. The results suggested that the major cause of HR-
$\mathrm{AD}+$ wasabi diet increase over the HR-AD diet was the increase in energy intake.

Effects of wasabi rhizome extract on scratching behavior

In HR-AD diet-fed HR-1 mice, nerve fibers markedly increased in the epidermis (24). The increase in intraepidermal nerve fibers, as well as the skin barrier dysfunction, plays a crucial role in the increased 
scratching behavior. We investigated the effects of intake of wasabi rhizome extract on scratching behavior (Fig. 1). HR-AD-fed mice indicated a significantly high value compared with that of normal diet-fed mice on days 28, 35 and 42. On the other hand, HR$\mathrm{AD}+10 \%$ wasabi diet-fed mice indicated significantly lower values compared with that of HR-AD-fed mice on days 28, 35 and 42, and compared with that of HR$\mathrm{AD}+5 \%$ wasabi diet-fed mice on day 35 . Thus, the wasabi-containing diet was highly effective in preventing scratching behavior in HR-1 hairless mice.

Effects of wasabi rhizome extract on the plasma components and histopathological indexes

In four of the plasma components (histamine, eotaxin, IgE and TARC), HR-AD diet-fed mice were significantly higher than normal diet-fed mice (Fig. 2). On the other hand, HR-AD $+10 \%$ wasabi diet-fed mice were significantly lower than HR-AD diet-fed mice in four of the plasma components. HR-AD $+5 \%$ wasabi diet-fed mice indicated decreasing but not significant tendency. In all of the histopathological indexes (T.B., MBP, CD4, IL-4, IL-5, eotaxin, TARC and IgE), HR-AD diet-fed mice were significantly higher than normal diet-fed mice (Fig. 3). In all of histopathological indexes, HR-AD $+5 \%$ and $10 \%$ wasabi diet-fed mice were significantly lower than HR-AD diet-fed mice. The wasabi rhizome extract-containing diet used in this study repressed scratching behavior and reduced the level of chemical mediators. It was considered that feeding of a wasabi rhizome extract suppressed cross talk between cells which participate in skin injury such as Th2 cells, B cells, mast cells and fibroblasts at several points of signaling. But the molecular target of the substance included in the wasabi rhizome extract was not clear.

In our study, the substance which was responsible for the improvement of the AD-like symptom was not clear. HR-1 hairless mice fed a diet with a reduced magnesium level developed AD-like symptom (22). The magnesium content of each diet was estimated at 2,700 $\mathrm{mg} / \mathrm{kg}$ in the normal diet, $200 \mathrm{mg} / \mathrm{kg}$ in the HR-AD diet, $370 \mathrm{mg} / \mathrm{kg}$ in the $\mathrm{HR}-\mathrm{AD}+5 \%$ wasabi diet and $560 \mathrm{mg} / \mathrm{kg}$ in the HR-AD $+10 \%$ wasabi diet. The accumulation of magnesium intake on measurement day (day 0, 7, 14, 21, 28, 35 and 42) was estimated at $112.9 \pm 3.7 \mathrm{mg}$ in the normal diet-fed mice, $5.7 \pm 0.2 \mathrm{mg}$ in the HR-AD diet-fed mice, $12.6 \pm 0.5 \mathrm{mg}$ in the HR-AD $+5 \%$ wasabi diet-fed mice and $17.9 \pm 0.6 \mathrm{mg}$ in the HR-AD $+10 \%$ wasabi diet-fed mice (mean \pm SE). The magnesium intake of HR-AD diet-fed mice and wasabi diet-fed mice were significantly lower than that of normal diet-fed mice. There was statistical significance between HR-AD-fed mice and wasabi dietfed mice. Akamatsu et al. made AD-like model mice using a special diet including magnesium of $1,400 \mathrm{mg} /$ $\mathrm{kg}(23,25)$. In our study, magnesium in wasabi rhizome extract is low; it appeared that magnesium derived from wasabi was not a key substance involved in the improvement of the AD-like symptoms.

Morimitsu et al. reported that a part of 6-MSITC entered the circulatory system as its glutathione conju- gate (26). Uto et al. reported that 6-MSITC inhibited MAPK signaling pathways in inflammation (19, 27). It has also been reported that expressions of TARC and eotaxin are controlled via MAPK signaling pathways (28, 29). 6-MSITC might adjust chemical mediators through MAPK signaling pathways. There is a possibility that 6-MSITC is the key substance which improved the AD-like symptoms in HR-1 hairless mice.

This study showed that wasabi rhizome extract might be efficacious in the treatment of human AD.

\section{REFERENCES}

1) Furue M, Saeki H, Furukawa F, Hide M, Ohtsuki M, Nakamura T, Sasaki R, Suto H, Takehara K. 2008. Guideline for management of atopic dermatitis. Nippon Hihuka Gakkai Zasshi 118: 325-342 (in Japanese).

2) Hoffman DR, Yamamoto FY, Geller B, Haddad Z. 1975. Specific IgE antibodies in atopic eczema. J Allergy Clin Immunol 55: 256-267.

3) Ring J. 1983. Plasma histamine concentrations in atopic eczema. Clin Allergy 13: 545-552.

4) Welle MM, Olivry T, Grimm S, Suter M. 1999. Mast cell density and subtypes in the skin of dogs with atopic dermatitis. J Comp Pathol 120: 187-197.

5) Inada N, Shoji J, Tabuchi K, Saito K, Sawa M. 2004. Histological study on mast cells in conjunctiva of $\mathrm{NC} / \mathrm{Nga}$ mice. Jpn J Ophthalmol 48:189-194.

6) Vestergaard C, Bang K, Gesser B, Yoneyama H, Matsushima K, Larsen CG. 2000. A $\mathrm{Th}_{2}$ chemokine, TARC, produced by keratinocytes may recruit $\mathrm{CLA}^{+} \mathrm{CCR} 4^{+}$ lymphocytes into lesional atopic dermatitis skin. J Invest Dermatol 115: 640-646.

7) Dworzak MN, Fröschl G, Printz D, Fleischer C, Pötschger U, Fritsch G, Gadner H, Emminger W. 1999. Skin-associated lymphocytes in the peripheral blood of patients with atopic dermatitis: signs of subset expansion and stimulation. J Allergy Clin Immunol 103: 901-906.

8) Miyamasu M, Nakajima T, Misaki Y, Izumi S, Tsuno N, Kasahara T, Yamamoto K, Morita Y, Hirai K. 1999. Dermal fibroblasts represent a potent major source of human eotaxin: In vitro production and cytokine-mediated regulation. Cytokine 11: 751-758.

9) Ring J, Bieber T, Vieluf D, Kunz B, Przybilla B. 1991. Atopic eczema, Langerhans cells and allergy. Int Arch Allergy Appl Immunol 94: 194-201.

10) Gleich GJ, Loegering DA, Kueppers F, Bajaj SP, Mann KG. 1974. Physiochemical and biological properties of the major basic protein from guinea pig eosinophil granules. J Exp Med 140: 313-332.

11) Kakinuma $T$, Nakamura $K$, Wakugawa M, Mitsui $H$, Tada Y, Saeki H, Torii H, Asahina A, Onai N, Matsushima K, Tamaki K. 2001. Thymus and activation-regulated chemokine in atopic dermatitis: Serum thymus and activation-regulated chemokine level is closely related with disease activity. J Allergy Clin Immunol 107: 535-541.

12) Fujisawa T, Fujisawa R, Kato Y, Nakayama T, Morita A, Katsumata H, Nishimori H, Iguchi K, Kamiya H, Gray PW, Chantry D, Suzuki R, Yoshie O. 2002. Presence of high contents of thymus and activation-regulated chemokine in platelets and elevated plasma levels of thymus and activation-regulated chemokine and macrophage-derived chemokine in patients with atopic dermatitis. J Allergy Clin Immunol 110: 139-146. 
13) Jahnz-Rozyk K, Targowski T, Paluchowska E, Owczarek W, Kucharczyk A. 2005. Serum thymus and activationregulated chemokine, macrophage-derived chemokine and eotaxin as markers of severity of atopic dermatitis. Allergy 60: 685-688.

14) Omoto M, Gu LH, Sugiura H, Uehara M. 2000. Heterogeneity of dermal deposition of eosinophil granule major basic protein in acute lesions of atopic dermatitis. Arch Dermatol Res 292: 51-54.

15) Isshiki K, Tokuoka K, Mori R, Chiba S. 1992. Preliminary examination of allyl isothiocyanate vapor for food preservation. Biosci Biotechnol Biochem 56: 14761477.

16) Ono H, Tesaki S, Tanabe S, Watanabe M. 1998. 6-Methylsulfinylhexyl isothiocyanate and its homologues as food-originated compounds with antibacterial activity against Escherichia coli and Staphylococcus aureus. Biosci Biotechnol Biochem 62: 363-365.

17) Fukuchi Y, Kato Y, Okunishi I, Matsutani Y, Osawa T. 2004. 6-Methylsulfinylhexyl isothiocyanate, an antioxidant derived from Wasabia japonica Matsum, ameliorates diabetic nephropathy in type 2 diabetic mice. Food Sci Technol Res 10: 290-295.

18) Morimitsu Y, Hayashi K, Nakagawa Y, Horio F, Uchida K, Osawa T. 2000. Antiplatelet and anticancer isothiocyanates in Japanese domestic horseradish, wasabi. Biofactors 13: 271-276.

19) Uto T, Fujii M, Hou D-X. 2005. 6-(Methylsulfinyl)hexyl isothiocyanate suppresses inducible nitric oxide synthase expression through the inhibition of Janus kinase 2-mediated JNK pathway in lipopolysaccharide-activated murine macrophages. Biochem Pharmacol 70: 1211-1221.

20) Kubo M, Kambayashi Y, Takemoto K, Okuda J, Muto M, Ogino K. 2005. Reactive nitrogen species formation in eosinophils and imbalance in nitric oxide metabolism are involved in atopic dermatitis-like skin lesions in $\mathrm{NC} /$ Nga mice. Free Radic Res 39: 719-727.

21) Taniuchi S, Kojima T, Hara Mt K, Yamamoto A, Sasai M, Takahashi H, Kobayashi Y. 2001. Increased serum nitrate levels in infants with atopic dermatitis. Allergy 56: 693-695.

22) Fujii M, Tomozawa J, Mizutani N, Nabe T, Danno K, Kohno S. 2005. Atopic dermatitis-like pruritic skin inflammation caused by feeding a special diet to HR-1 hairless mice. Exp Dermatol 14: 460-468.

23) Akamatsu H, Makiura M, Yamamoto N, Yagami A, Shimizu Y, Matsunaga K. 2006. The effect of fexofenadine on pruritus in a mouse model (HR-ADf) of atopic dermatitis. J Int Med Res 34: 495-504.

24) Fujii M, Akita K, Mizutani N, Nabe T, Kohno S. 2007. Development of numerous nerve fibers in the epidermis of hairless mice with atopic dermatitis-like pruritic skin inflammation. J Pharmacol Sci 104: 243-251.

25) Makiura M, Akamatsu H, Akita H, Yagami A, Shimizu Y, Eiro H, Kuramoto M, Suzuki K, Matsunaga K. 2004. Atopic dermatitis-like symptoms in HR-1 hairless mice fed a diet low in magnesium and zinc. J Int Med Res 32: 392-399.

26) Morimitsu Y, Nakagawa Y, Hayashi K, Fujii H, Kumagai T, Nakamura Y, Osawa T, Horio F, Itoh K, Iida K, Yamamoto M, Uchida K. 2002. A sulforaphane analogue that potently activates the Nrf2-dependent detoxification pathway. J Biol Chem 277: 3456-3463.

27) Uto T, Fujii M, Hou D-X. 2005. Inhibition of lipopolysaccharide-induced cyclooxygenase- 2 transcription by 6(methylsulfinyl)hexyl isothiocyanate, a chemopreventive compound from Wasabia japonica (Miq.) Matsumura, in mouse macrophages. Biochem Pharmacol 70: 1772-1784.

28) Heijink IH, Marcel Kies P, van Oosterhout AJ, Postma DS, Kauffman HF, Vellenga E. 2007. Der p, IL-4, and TGF- $\beta$ cooperatively induce EGFR-dependent TARC expression in airway epithelium. Am J Respir Cell Mol Biol 36: 351-359.

29) Wong CK, Zhang JP, Ip WK, Lam CW. 2002. Activation of p38 mitogen-activated protein kinase and nuclear factor-kappaB in tumour necrosis factor-induced eotaxin release of human eosinophils. Clin Exp Immunol 128: $483-489$. 